\title{
En-Route Vehicular Traffic Optimization
}

\author{
Saravanan M \\ Ericsson Research India \\ Ericsson India Global Services Pvt. Ltd \\ Chennai, India
}

\author{
Ashwin Kumar M \\ Dept. of Electronics and Communication Engineering \\ Meenakshi Sundarajan Engineering College \\ Chennai, India
}

\begin{abstract}
The pathways of information are changing, the physical world itself is becoming a type of information system. In what's called the Internet of Things (IoT), sensors and actuators embedded in physical objects - from roadways to pacemakersare linked through wired and wireless networks, often using the same Internet Protocol (IP) that connects the Internet. When objects can both sense the environment and communicate, they become tools for understanding complexity and responding to it swiftly. The revolutionary part in all this is that these physical information systems are now beginning to be deployed, and some of them even work largely without human intervention. This paper has addressed the traffic congestion problem with the help of Internet of Things. Increase in the number of vehicles in cities caused by the population and development of economy, has stimulated traffic congestion problems. It is becoming more serious day after day in the present scenario of developing countries. The reason for the same could be categorized as mismanagement of vehicular movement, ineffective system for controlling the mobility of vehicles, uneven roads and traffic snarl-up. Unexpected vehicular queuing is a major concern leading to wasting time of passengers and thwarting ambulance to reach the destination in time. In addition to that, traffic congestion makes it difficult to forecast the travel time accurately causing drivers to allocate more time in travel than scheduled previously. To ease these mounting traffic problems a demonstration is made on the Proof of Concept (POC) using the smart city data set provided by Telecom Italia of Milan city, to verify that these concepts have the potential for real world application and could be used by the government sectors or private transport organizations to ameliorate the passenger's comfort on road which are as follows. A central node is developed which sets the speed limit and predicts a normalized speed separately for each locality from the available data set. For efficient control in mobility of vehicles an advanced dynamic digital board is introduced, which displays the speed limit set by the central node time to time. The normalized speed could be used to estimate the effective time taken between destinations precisely. By comparing normalized speed with real time values anomalies in the locality like congestion and presence of uneven roads is predicted. Accident detection model is integrated with the central node which sends a message to dynamic board indicating location of the accident along with the time taken. It even improves traffic flow around the accident occurred location.
\end{abstract}

Central node together with navigation tools could provide rerouted path to the drivers during congestion or accident.

Keywords-IoT; IP; POC; Central Node; Dynamic Board; Accident detection model

\section{INTRODUCTION}

Machine to Machine (M2M) communication along with its relevance IoT (Internet of Things) technology is simple, embedded and invisible to connect billions of devices to introduce Networked Society [1]. Many cities and homes are changed to the new norm of smart in the sense that all the devices attached are interconnected and communicated with each other for the benefit of the society uplifting. The connected device details of one of a smart city are analyzed and reported in this paper.

Traffic congestion is a major concern to all countries, be it, developing or developed. Unexpected vehicular queuing is a major concern leading to wastage of time of passengers and thwarting of ambulance to reach the destination in time. Adding to that, traffic congestion makes it difficult to forecast the travel time accurately causing drivers to spend more time in travel than scheduled previously. The idea is to optimize traffic congestion solutions with the help of existing intelligent sensor devices. These devices can measure many parameters for a more efficient route management of the city. The remote sensor gathers data and sends it wirelessly to a network, where it is next routed, often through the Internet (with IoT facility), to a server such as a designated node. At that point, the data is continuously recorded, analyzed and acted upon the connected devices with less human intervention. The block diagram (Fig 1) shows the integrated view of various components built in the proposed system. Here central node acts like a hub in receiving messages from sensors and vehicles during an accident and communicates this with the dynamic board by sending the message it has to display. The main contributions of this work are to address the real issues pertaining to vehicular traffic optimization in a smart city. 


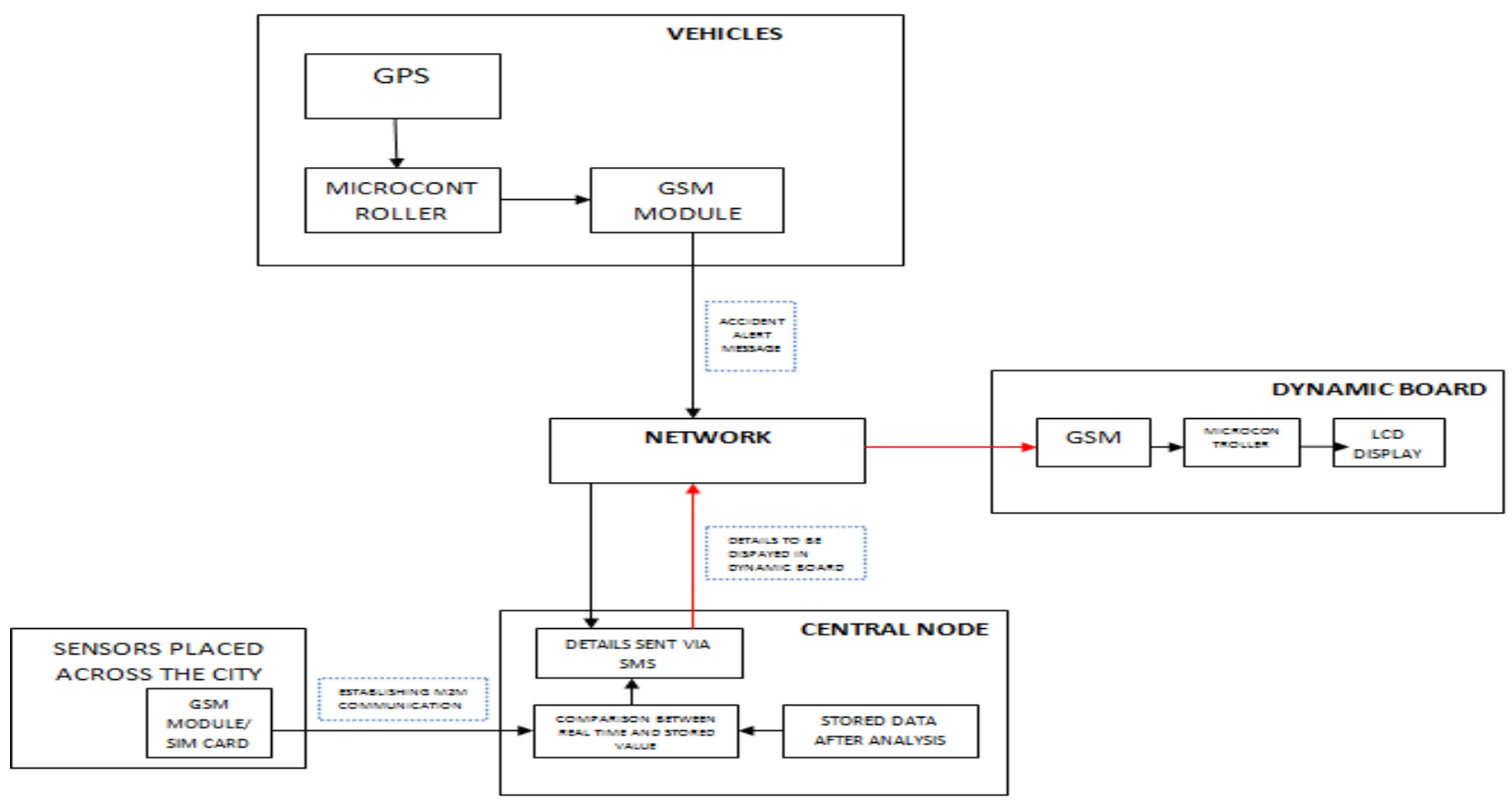

Fig. 1. Block diagram. Central node acts like a hub receiving messages from sensors and alert messages directly from the vehicles during accident and communicates with the dynamic board by sending messages it has to display. GSM is used for sending and receiving messages, black and red arrows are used to differentiate receiving and sending messages respectively

\section{A. Management of vehicular movement}

The central node is introduced, which predicts the speed limit across the city in an hourly basis considering people's movement and traffic flow in a locality and instantaneously varying the speed limit during accidents and unexpected traffic snarl up. Dynamic board is constructed and setup in various places across the city which displays the speed limit predicted by the central node, thereby ensuring efficient control in mobility of vehicles. Along with speed limit, central node predicts normalized speed (median speed) separately for each locality from the available data set. This normalized speed is used to estimate time taken between destinations which help commuters to schedule their travel plan more efficiently.

\section{B. Traffic snarl-up}

The reasons for traffic snarl-up are unexpected congestion and also the occurrence of accidents. In order to prevent unexpected congestion the rerouted path is provided to the user with the help of navigation tools and dynamic boards. In order to reduce the traffic during accidents in a particular area, accident detection model is integrated with the central node which alerts the nearby hospitals immediately and sends a message to dynamic board indicating location of the accident along with the time taken for ambulance to cross the board through Global System for Mobile (GSM) module. This would avoid congestion around the accident location and also ensure ambulance to reach the destination in time.

\section{Uneven road}

By comparing normalized speed with real time inductive loop (a device laid on the road within certain range) values, any anomalies in the locality like congestion and presence of uneven roads could be predicted by understanding the change in speed of the vehicles.

\section{RELATED WORK}

Traffic congestion problem had numerous solutions in the past. There have been multiple systems which address specific parts of the problem but lacked to provide end-toend solutions and faced many difficulties to prove its effectiveness. Here discussions about few such systems along with its limitations are made. The existing works of predicting the speed limit is based on peak hour traffic and weather changes [2]. The concept of offline routing exists. A particular node speed limit value is set only based on the available data of peak hour traffic, static speed limit and change to another static value during the presence of rain or other reasons. Its occurrence is detected with the help of rain sensors equipped in speed board. This leads to frustration of travelers when they have to abide to the speed limit set based on peak hour traffic during night hours. In addition to this, fixed speed limit leads to lack of significant control over mobility of vehicles. Even in some developed countries, variable speed boards have been designed with only few parameters being taken into considerations like lightning and construction zone [3]. This shows there is a lack of efficient method to set the speed limit, which ultimately leads to mismanagement of vehicular movements.

Another system includes a Global Positioning System (GPS) based navigational system that includes a GPS receiver connected to a wireless communication device for communicating with a remote computer over a wireless communication network [4]. The GPS-based navigation system continuously determines the motored vehicle's exact 
physical location in a region that is intermittently or continuously uploaded to a remote computer via the wireless communication network, this work aids at finding the traffic congestion in a given area. But the problem is during such huge traffic the remote computer faces certain difficulties to manage the details of the each GPS receiver. Monitoring such a huge traffic of data is very expensive.

Various sensors such as 3D laser scanning devices along with $3 \mathrm{D}$ reconstruction algorithms are used to measure the size of road potholes [5, 6, 7]. In [8, 9], cameras are installed on vehicles to record road videos, from which road conditions are inferred. With the help of Accelerometer, vibrations on the road can be effectively captured from the vehicles and along with GPS, its actual location is determined [10]. The system to detect the presence of uneven roads uses 3D laser scanning devices or cameras to record images and videos or Accelerometer plus GPS equipped inside a car, but all these three systems are too expensive for wide adoption and communication from a car to a remote control stills remains a challenging task as mentioned earlier.

Some research studies were carried out on accident detection system, aerial surveillance or close circuit surveillance [11]. All these systems are used to determine accident but the drawback of this system is that, someone has to witness and report the accident. When severity of the accident is high, the driver reporting accident system fails, as the driver may not be able to report in such conditions. Smart phone based accident detection models [12] are very expensive and even lead to false alarm. Continuous monitoring of vehicular GPS device through a remote computer help to detect accident instantaneously but monitoring the huge traffic of data is challenging [4]. Researchers have also reduced the traffic by sending only alarm to remote computer during accident occurrence with the help of microcontroller, GPS and GSM equipped vehicles [13] but still it lacks end-to-end results because it lacks efficient rescue operations.

Another typical problem is to predict the accurate travelling time in advance which is mostly required for the specific industry need and people's daily life. Researchers have come up with some systems for estimating efficient time taken. Travel time prediction algorithms attempt to estimate the time of travel between an arbitrary origin-destination pair in a roadway network, for current and future instances. It assists the driver to choose a less congested route, thus can be used for optimal routing and dynamic route guidance $[14,15]$. There is also a model to estimate the travel time that begins at a long-term future moment of departure [16]. This system lacks real estimation of time, because several factors like current traffic, congestion due to occurrence of big events, presence of accident and so on are not taken into considerations. In this paper, we are addressing these challenges to bring out a better system to optimize and improve the present smart city environment.

\section{DATASET DESCRIPTION}

At present, data is woven into every sector and function in the global economy. The use of Big Data Analytics - large pools of data that can be brought together and analyzed to discern patterns to make better decisions in enhancing productivity, creating significant value for the world economy by reducing waste, increasing the quality of products and thereby improving customer services. Telecom Italia on January 2014 released different categories of data like telecommunication, energy, weather, health insurance company details, public and private transport, Social Networks and events to the international scientific community. The data provided within the dataset of the Big Data Challenge will be geo-referenced and anonymous relating to the territories of Milan and of the Autonomous Province of Trento [17]. The dataset contains millions of records of data covering the period from November to December 2013.

B y utilizing the dataset provided by Telecom Italia [17] and by categorizing city details into referred grids (each square id of area $235^{*} 235 \mathrm{~m}^{2}$ ), the people movement in a grid is estimated with the help of available call detail records. For predicting median speed (normalized speed) in a grid, the inductive loop detectors (sensors installed across city providing details of number of cars crossing the detector and speed of each car along with its direction) are used. The private insurance company has installed GPS device inside a car for its customers. Using this, the traffic data is obtained and it also provides details of number of users in each grid along with the average speed.

\section{SYSTEM OVERVIEW}

The main components of the system include central node, dynamic board and accident detection system in vehicles. Available data are processed and stored in central node and real time communication between central node and various sensors like inductive loop detector, precipitation sensor and vehicles-accident detection model is established. With the help of GSM module, central node communicates with the digital board via Short Message Service (SMS) regarding details it must display. These digital boards are called as dynamic board in this paper, as it displays the streaming of information on a continuous basis. Detailed explanation of each of the attached component is given below.

\section{A. Dynamic Board}

Dynamic boards are used only to receive text from central node and display the speed limit, location of accident and time taken for the ambulance to cross. Each board composed of Liquid Crystal Display (LCD) screen to display the message, GSM module for receiving messages and a microcontroller which acts like an interface between the two.

1) GSM module - It is used to receive the information (SMS) from the central node. The information received from the central node is displayed in the board. Normally speed limit will be sent from central node and incase of accident, its location and time taken for the ambulance to cross the dynamic board will be provided.

2) Microcontroller - It acts like an interface between GSM module with LCD Display, it is coded to receive the information from a particular mobile number (central node) and display it in the central node. 
3) LCD Display - This electronic display is used as a communication medium between the central node and regular commuters of the route. Dynamic board is just a device which receives details and displays it in the display.

\section{B. Central Node}

An Intellect Central Node is a remote monitoring system used for storing the processed data. In addition to that, it receives information from all the available sensors. It compares the real time values along with the predicted values to find if any anomalies are present and during the case of anomalies it takes the necessary steps needed to control the situation. In this study, anomalies are considered as the occurrence of an accident, reduced speed limit in a grid (uneven roads), drastic increment in call detail record value (increase in people movement) and presence of rain (weather sensor). Central node sends the details it should display in an hour interval, but recognising any anomalies in a grid it immediately sends new details, board must display and intimate necessary details along with it.

\section{Vehicles}

Vehicles act as an accident detection device, it makes use of GPS receiver, Microcontroller and GSM module while GPS receiver tracks all the satellites and provides accurate positioning data in NMEA (National Marine Electronics Association) standards, GPRMC (Recommendation minimum data for GPS) sentences (which recommends minimum specific GPS/Transit data) in every second [17]. In Microcontroller, memory spaces are allocated for speed and position (latitude and longitude), and values are separated and stored by interpreting the position in GPRMC sentences with the help of the number of commas. The Microcontroller unit checks the variation in speed for every second, by comparing it with the previous value. If the variation is more than or equal to $28.8 \mathrm{kph}$ for $1 \mathrm{sec}$ then, the microcontroller expects the traveller to respond if it's a false detection and waits for 5 seconds. If the traveler fails to respond then accident alert is triggered and sent to central node using GSM module. Speed variation concept (variation of speed for detecting accident) is explained under Section 7.

\section{TOOLS OVERVIEW AND ACTIVITY}

MATLAB [18] and LABVIEW [19] software tools were used to develop the simulated model and a brief description about the activities are given here.

\section{A. Tool- based Activities}

For the year 2009-2013, vehicles in operation (per 1000) in Italy was 682 and the numbers are increasing day-by-day, it creates a scenario of monitoring or controlling the vehicle movements which as become a strenuous job. In our implementation, all the discussed difficulties and design activities for an efficient management and controlling of vehicle movements are considered. For each activity, concerned components usage is listed out and brief explanation is provided along with its effectiveness for the requirements.

1) Display in Dynamic speed board: Dynamic boards are set up across the city to display speed limit dynamically which in turn help in restricting the speed of vehicle movements and hence controlling the mobility of vehicles in operation. The idea of dynamic board replacing the normal static board is to enhance its effectiveness. That is in static boards speed limit remains the same during the peak hours and midnight resulting in frustration of the travelers. This is overcome by predicting the speed limit based on traffic flow and people movement from the available data and displaying the same. In addition to it, the flow of the vehicles is controlled during abnormalities like accident and sudden increase in people movement in specific location by normalizing the display in speed limit (display dynamically) and thus ensure proper movement of vehicles during crisis. Since the speed limit is set based on the model learned from the previous year data in a grid, accuracies of its prediction remains high and law enforcement could be tightened.

2) Rerouting: Occurrence of accident leads to traffic snarl-up. The best way to prevent this would be by rerouting and minimizing the vehicle movements. With the available navigation tool the concept of rerouting is included. The predicted shortest path for the user is based on the time taken between places. The travel time duration for vehicles is optimized during accidents. Instead of normalized speed, reduced speed limit is taken into consideration. This allows travel time duration to increase considerably and thus the system avoids the particular node (accident location) and provides the shortest path by considering the travel duration.

3) Congestion avoidance: Avoidance during congenstion is managing the flow of vehicles during abnormalities like accidents, sudden increase of people movement in certain location. When central node is alerted about the same with the help of latitude and longitude provided by the GPS inside a car that predicts the accident grid and also its neighboring grids and sends the optimized speed limit message to the same along with its accident occurred location. This provides knowledge about the congested area to the travelers well in advance and would preferably guide them to avoid the route if possible.

4) Detecting uneven roads: Presence of uneven roads is always hindrance to the travelers and installation of sensors for complete monitoring of all roads will not be possible. Here we make use of the available sensors and design a cost effective model. The main idea behind this model is presence of uneven roads would lead to reduced speed of vehicles. By understanding the current real time situation and map it along with the available data of previous year to predict uneven roads. Possible situations could be changed in weather patterns, people movement and traffic flow during government holidays and normal days and so on.

\section{DATA ANALYSIS}

The analysis of the telecom Italia of Milan city data constitutes integral part of this model. The telecom data are being grouped into seven types which are listed in Table 1 . Based on weather phenomenon the predicted value is being stored in modules after performing mathematical 
calculations. In each module speed limit, normalized speed, traffic flow and people movement are predicted and stored for each hour separately indicated in Table 2, for all the Square ids (City of Milan is sub divided into 10,000 square ids with area $235 * 235 \mathrm{~m}^{2}$, in this paper we consider each square id has different locality). Module name being the square id number followed by $\mathrm{R}$ (rain) or $\mathrm{A}$ (absent) or I (immediate) indicating the weather conditions (i.e.) 2260A1 or 2260I1 or 2260R1.

The available data are being categorized under the following category

- Tuesday to Thursday (type 1)

- Friday (type 2)

- Saturday (type 3)

- Sunday (type 4)

- Monday(type 5)

- Unexpected increase in traffic (type 6)

- Unexpected increase in people movement (type 7)

In this section, few relevant variables have been predicted. This could be useful to implement the activities required to address the traffic congestion problems.

TABLE I. GRouping OF DATA Module BASED ON 7 TyPES

\begin{tabular}{|l|l|l|l|l|}
\hline Types & \multicolumn{1}{|l|}{$\mathrm{Z}=0$} & \multicolumn{2}{l|}{$\mathrm{Z}=1$} & $\begin{array}{l}\mathrm{Z}=2(\mathrm{Imm} \quad \text { after } \\
\text { rain/snow) }\end{array}$ \\
\hline & & Rain & Snow & \\
\hline Type 1 & $2233 \mathrm{~A} 1$ & $2233 \mathrm{R} 1$ & $2233 \mathrm{~S} 1$ & $2233 \mathrm{I} 1$ \\
\hline Type 2 & $2233 \mathrm{~A} 2$ & $2233 \mathrm{R} 2$ & $2233 \mathrm{~S} 2$ & $2233 \mathrm{I} 2$ \\
\hline Type 3 & $2233 \mathrm{~A} 3$ & $2233 \mathrm{R} 3$ & $2233 \mathrm{~S} 3$ & $2233 \mathrm{I} 3$ \\
\hline Type 4 & $2233 \mathrm{~A} 4$ & $2233 \mathrm{R} 4$ & $2233 \mathrm{~S} 4$ & $2233 \mathrm{I} 4$ \\
\hline Type 5 & $2233 \mathrm{~A} 5$ & $2233 \mathrm{R} 5$ & $2233 \mathrm{~S} 5$ & $2233 \mathrm{I} 5$ \\
\hline Type 6 & $2233 \mathrm{~A} 6$ & $2233 \mathrm{R} 6$ & $2233 \mathrm{~S} 6$ & $2233 \mathrm{I} 6$ \\
\hline Type 7 & $2233 \mathrm{~A} 7$ & $2233 \mathrm{R} 7$ & $2233 \mathrm{~S} 7$ & $2233 \mathrm{I} 7$ \\
\hline
\end{tabular}

\section{A. Normalized Speed}

The available speed values for different vehicles are stored based on weather conditions and extreme values are eliminated from the available set. The median value of the available set for the particular time interval is taken as a normalized speed. Inductive loop sensor and private insurance company details are being used for predicting normalized speed.

\section{B. People Movement}

Number of people in a locality is estimated based on CDR(call detail record ) details. SMS IN, SMS OUT, CALL IN, CALL OUT and DATA PACK values are grouped together to estimate the approximate estimate of people in a locality. The movement of people is estimated through relative ranking mechanism (i.e.) highest number of CDR's generated in a certain location for specific time period will be considered as highest values (for ex. 100) and the relative percentage is worked out for the remaining locations related to each square id.

\section{Traffic Flow}

The traffic flows are being grouped under types shown in Table 1 based on weather conditions and saved as module wise. The traffic flow strength in each square id is estimated similarly to people movement which is based on relativity.

\section{Speed limit}

The details from Italian Highway Code (Codice della Strada) of speed limit for several categories of roads like urban roads, motorways and so on are extracted. The available data is sandwiched along with the speed limit details to predict the node speed limit based on the traffic flow and people movement.

TABLE II. STORED REPRESENTATIONS OF VARIOUS PARAMETERS

\begin{tabular}{|l|l|l|l|l|}
\hline $\begin{array}{l}\text { Time } \\
\text { Interval }\end{array}$ & $\begin{array}{l}\text { Normalized } \\
\text { Speed }\end{array}$ & $\begin{array}{l}\text { Traffic } \\
\text { Flow }\end{array}$ & $\begin{array}{l}\text { People } \\
\text { Movement }\end{array}$ & $\begin{array}{l}\text { Speed } \\
\text { Limit }\end{array}$ \\
\hline 00:00-01:00 & $110 \mathrm{~km} / \mathrm{hr}$ & 32 & 104 & $130 \mathrm{~km} / \mathrm{hr}$ \\
\hline $01: 00-02: 00$ & $90 \mathrm{~km} / \mathrm{hr}$ & 23 & 167 & $110 \mathrm{~km} / \mathrm{hr}$ \\
\hline$:$ & $:$ & $:$ & $:$ & $:$ \\
\hline$:$ & $:$ & $:$ & $:$ & $:$ \\
\hline $22: 00-23: 00$ & $60 \mathrm{~km} / \mathrm{hr}$ & 16 & 123 & $90 \mathrm{~km} / \mathrm{hr}$ \\
\hline 23:00-00:00 & $90 \mathrm{~km} / \mathrm{hr}$ & 13 & 157 & $110 \mathrm{~km} / \mathrm{hr}$ \\
\hline
\end{tabular}

VII. IMPLEMENTATION OVERVIEW

Detailed explanation for implementing the activities in MATLAB [18] and LABVIEW [19] is provided in this section.

\section{A. Effective Time Estimation}

In the existing time estimation models, approximated speed value is set for a long range of distance and variation of that traffic is not regularly updated. Both the problems are solved by breaking long range of distance into several short range distance groups based on the number of bends. The speed value is provided for each short distance range via Inductive loop detector (if loop sensor falls in the path) or private insurance company details. The average speed value is derived for the particular grid id and the time taken is estimated for each time interval. The node's latitude and longitude are used to estimate the distance value for short range distance groups separately with the help of Haversine formula [20]. Similarly nodes of four different routes are considered for developing a sample map considering small part of Milan, all the edges and junctions in route are set as nodes. The path between two nodes is considered as distance pair and the distance is estimated via Haversine formula. For each distance pair the details about their corresponding grid id is stored. These nodes are assigned names based on the location and with the help of MATLAB, Dijkstra's algorithm [18] is run to find the shortest path between the destinations. Since no negative edges are being used, the 
Dijkstra's algorithm is ideal to find the shortest path from the sample map.

$a=\sin \frac{\nabla \varphi}{2} \sin \frac{\nabla \varphi}{2}+\cos \varphi 1 \cos \varphi 2 \sin \frac{\nabla \kappa}{2} \sin \frac{\nabla \kappa}{2}$

$c=2 \cdot a \cdot \tan 2(\sqrt{a}, \sqrt{ }(1-a))$

$d=R . c$

where $\varphi$ is latitude, $\mathbb{N}$ is longitude, $\mathrm{R}$ is earth's surface ( mean radius $=6371 \mathrm{~km}$

\section{B. Accident Detection}

Accident is detected through vehicle movements by monitoring the speed variation. It is predicted that if the speed variation is equal to or greater than $28.8 \mathrm{~km} / \mathrm{hr}$ for a second, then there might be an accident or abrupt halt of vehicles irrespective of its speed. The two different initial speeds vary between $90 \mathrm{~km} / \mathrm{hr}$ and $110 \mathrm{~km} / \mathrm{hr}$. The respective final velocities are $61.2 \mathrm{~km} / \mathrm{hr}$ and $81.2 \mathrm{~km} / \mathrm{hr}$. which is derived with the help of the elementary physics equation

$$
\mathrm{v}=\mathrm{u}-\mathrm{a} * \mathrm{t}
$$

It is understood that the difference between the initial and final velocities as $28.8 \mathrm{~km} / \mathrm{hr}$ in both the cases $(90-61.2,110$ 81.2).

\section{Dynamic speed board}

Dynamic speed board utilises the data related to speed limits stored in central node. These boards are installed at various locations across the city should be used as a device to communicate. In this study, the Milano grid number is used as the reference number for the dynamic board, as explained earlier the data is processed separately for each locality (for each square id) and central node is pre-programmed to send speed limit for every hour interval. For efficient control on the mobility of vehicles, the dynamic boards must be set up in the various junctions and lanes where the vehicle movements are high. Since the data provided to us are inductive loop detector values, it could be more advantageous and effective if dynamic boards are set up near these detectors. The weather phenomenon is unpredictable, weather monitoring system in a city immediately send a message to central node about the same and central node sends a message about the new speed limit immediately. GSM receiver is used in the dynamic board which receives the speed limit as a message and protocols are written which reads and writes the message (speed limit) in the LCD display.

\section{Traffic congestion avoidance}

During the time of accident, the GPS coordinates are received from accident detection module inbuilt in vehicles and based on the available latitude and longitude the grid id is predicted. The nearby grid id is chosen and the alert message is sent from the central node to the dynamic board indicating the change in speed limit, alerting the traveler the location of the accident. This helps local people to change the route on their own which would avoid congestion near the accident location. The shortest time taken path between the hospital and accident location is predicted and time estimation model is used. The source is from nearby hospital and destination is each dynamic board in the ambulance route (location of both is already stored in the central node). The time taken for ambulance to cross the dynamic board is estimated and it is sent as a message along with the speed limit and accident location. Thus dynamic board displays speed limit, accident location and time taken for ambulance to cross the particular dynamic board if it falls in the ambulance route. The idea is represented in Fig 2.

\section{E. Detecting uneven roads}

The normalized speed value is used to predict presence of uneven roads, the real time inductive loop values are compared along with the predicted normalized speed value based on the available inductive loop values as explained earlier. If the variation of the speed is more than $30 \%$ it means anomalies are predicted in a particular locality. The anomalies could be

(4)

- Accident

- Occurrence of an event in or Around the locality

- Presence of uneven roads

For avoiding the other 2 possibilities if the variations are existing for more than a day are checked. On the assumption that event doesn't occur for more than a day and also compare real time people movement and traffic flow for the particular square id with the predicted people movements and traffic flow. If the variation between these elements are found to be very high (threshold value for the variation is set to be 30 percentage) then it implies that people movement is increased in the locality and results in reduction of speed and if the variation is less, it shows the people movement in the locality is the same as earlier predicted and hence this leads to possibility of uneven road. Thus the roadway authorities can be informed on the same by using the details provided under administrative region.

\section{F. Rerouting}

Rerouting is simply suggesting the backup path when the primary path fails, here occurrence of accident or unexpected congestion in a locality leads to failure of the primary path. Occurrence of accident is detected with the help of GPS and GSM inside a car. The accident detection model was explained earlier in system overview. Unexpected congestion is determined with the help of CDR details which predicts number of people. Any huge increase in number of people is considered as congestion and a rerouted path is suggested to the user. For implementation the same sample map created earlier for the time estimation model is used. 


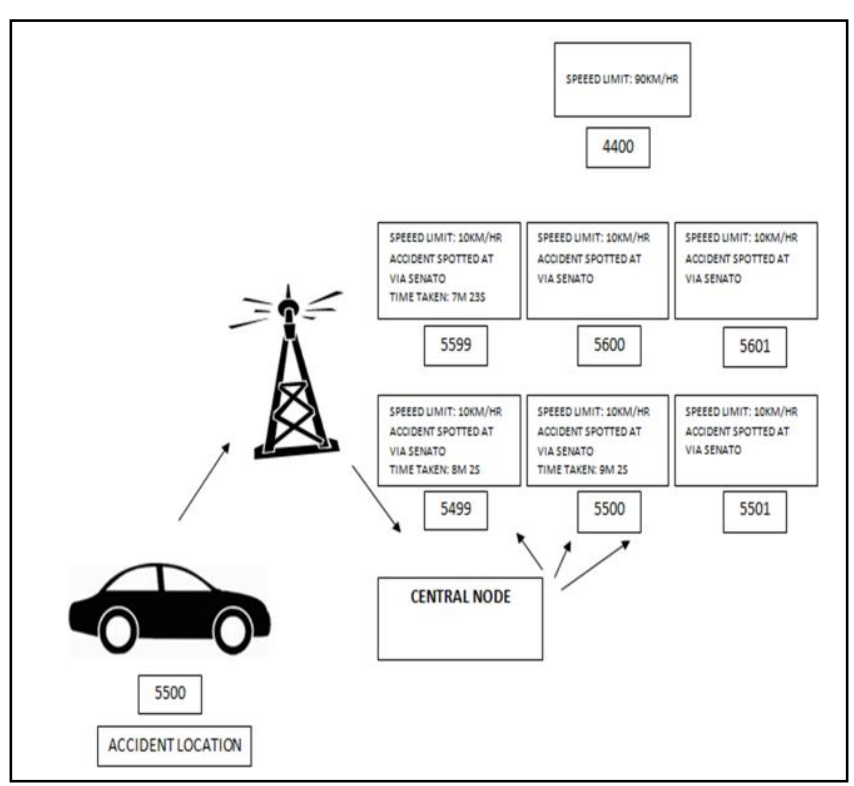

Fig. 2. Traffic congestion avoidance during accident. When central node is intimated about the accident directly from the vehicles, it finds the square id in which accident occurs with the help of latitude and longitude provided to them by the GPS inside car. Central node also picks the nearby square id and sends the message of new speed limit, accident location and time taken for the ambulance to cross the particular dynamic board (if the square id falls in the ambulance route) as shown in square id 5500 and sq id 4400 is far away from the accident location and hence speed limit doesn't varies

The rerouting concept is achieved by following steps.

a) Time taken is assigned as weights between nodes, in the form of distance by time.

b) If accident or any unexpected congestion is faced, central node allocates speed limit as $10 \mathrm{~km} / \mathrm{hr}$ which is updated in navigation tool between that particular nodes.

c) This implies time taken between the particular nodes will increase and subsequently the particular path is avoided by the navigation tool and alternative path is suggested.

\section{RESULTS AND DISCUSSION}

In data analysis, as mentioned earlier, the available data is processed and stored in an hourly basis, separately for every square id. The parameters are traffic flow, approximate number of people, normalized speed and speed limit. Fig 3A shown here is analysis of output for square id 2266. It can be inferred that, when people's movement and traffic increases, the speed limit drops down. During midnight, the speed limit stays high and it toggles from 70 to 45 during peak hours indicating the betterment and more effectiveness of the dynamic speed board. A plot to illustrate variations in speed limit based on traffic flow and people movement is shown in Figure 3B. Estimating effective time between 2 destinations requires a sample map and shortest path between users chosen directions for our estimation. A Google map [21] is used for comparison. Comparison between Google's predicted time and the model prediction is shown in Fig 4. But time predicted by the model is more efficient only when a good number of Inductive loop sensors are used rather than private insurance company details since the details provided by them are based on fewer number of vehicles. The requirement of GPS installed in vehicles being mandatory on these days, dependency on private insurance company details will also increase.

Traffic congestion model is similar to controlling the mobility of vehicles from the center. Several papers have been published under this domain and few have been implemented too and this promises to be future in traffic avoidance. Here only a single case either accident or occurrence of big event which leads to unexpected traffic snarl up is considered. A solution is provided for the same by controlling the flow of vehicles with the help of dynamic board and rerouting vehicles through navigation tools. With the help of LabView [19], an automated environment is simulated. When accident is spotted, alert is sent to the central node and it sends details about new speed limit, accident location information and time taken to cross the dynamic board if it lies in ambulance route to all nearby square ids. The representation of city in square id has eased the difficulties in spotting the nearby square id and hence the dynamic board. The screenshot of traffic congestion avoidance model is shown in Fig 5. This helps in controlling the flow of vehicles and it also helps the ambulance to reach the destination (for eg. accident spot) in time.

Only one way route is considered because this would let victim to get first aid access more quickly. Adding to that, rerouted path helps in reducing the vehicles queuing and ultimately reduces the traffic snarl up around accident location. The simulation of sample scenario using MATLAB [18] is givens in Fig 6.

\section{CONCLUSION}

An intellect central node along with dynamic speed board helped in addressing problems like proper management of vehicle movements, effective system for controlling the mobility of vehicles and understanding uneven roads and traffic snarl-up. Our proposed dynamic board set up at various locations in a city limits the vehicular speed and ensures control over vehicle's mobility. By considering all the real time parameters like traffic, weather or other anomalies, the efficient travel time between the destinations is estimated, which will help the user or the traveler to schedule plans accordingly. Vehicles are efficiently managed during anomalies like accidents by controlling the flow of vehicles, by reducing the speed limit and rerouting the local travelers by displaying the location of the accident in the dynamic board and by showing the rerouted path for other travelers using the navigation tool. To reduce the time taken for ambulance to reach the required destination the time taken for the ambulance to cross the dynamic boards is displayed in our approach. By displaying the time it is easy for the travelers to make way for the ambulance when necessary. Presence of uneven roads is always a hindrance to the travelers and halts the regular flow of the vehicles. With the help of the available sensors and M2M communication a cost efficient model is designed in this paper to predict the presence of uneven roads and report to the roadway authorities. 


\begin{tabular}{|c|c|c|c|c|c|c|c|}
\hline \multirow[t]{13}{*}{ D } & \multicolumn{6}{|l|}{ Command Window } & \multirow{2}{*}{ (2) } \\
\hline & $\gg$ analysis2 & & & & & & \\
\hline & 2.2660 & 0 & 0.0062 & 0 & 0.0779 & 0.1000 & \\
\hline & 2.2660 & 0.0010 & 0.0131 & 0 & 0.0747 & 0.1000 & \\
\hline & 2.2660 & 0.0020 & 0.0064 & 0.0028 & 0.0701 & 0.1000 & \\
\hline & 2.2660 & 0.0030 & 0.0052 & 0 & 0.0764 & 0.1000 & \\
\hline & 2.2660 & 0.0040 & 0.0058 & 0 & 0.0859 & 0.1000 & \\
\hline & 2.2660 & 0.0050 & 0.0082 & 0 & 0.0859 & 0.1000 & \\
\hline & 2.2660 & 0.0060 & 0.0291 & 0.0002 & 0.0849 & 0.0700 & \\
\hline & 2.2660 & 0.0070 & 0.0907 & 0.0548 & 0.0574 & 0.0500 & \\
\hline & 2.2660 & 0.0080 & 0.1039 & 0.1927 & 0.0164 & 0.0500 & \\
\hline & 2.2660 & 0.0090 & 0.1063 & 0.2775 & 0.0187 & 0.0400 & \\
\hline & 2.2660 & 0.0100 & 0.0917 & 0.3304 & 0.0332 & 0.0400 & \\
\hline 7 & 2.2660 & 0.0110 & 0.0737 & 0.3546 & 0.0591 & 0.0400 & \\
\hline \multirow{8}{*}{$\equiv$} & 2.2660 & 0.0120 & 0.0767 & 0.2714 & 0.0555 & 0.0500 & \\
\hline & 2.2660 & 0.0130 & 0.0698 & 0.2547 & 0.0623 & 0.0500 & \\
\hline & 2.2660 & 0.0140 & 0.0743 & 0.2834 & 0.0594 & 0.0450 & \\
\hline & 2.2660 & 0.0150 & 0.0798 & 0.2333 & 0.0575 & 0.0500 & \\
\hline & 2.2660 & 0.0160 & 0.0768 & 0.2570 & 0.0587 & 0.0500 & \\
\hline & 2.2660 & 0.0170 & 0.0704 & 0.1915 & 0.0581 & 0.0600 & \\
\hline & 2.2660 & 0.0180 & 0.0813 & 0.1165 & 0.0527 & 0.0500 & \\
\hline & 2.2660 & 0.0190 & 0.0729 & 0.0808 & 0.0539 & 0.0500 & \\
\hline \multirow{4}{*}{ A } & 2.2660 & 0.0200 & 0.0602 & 0.0613 & 0.0570 & 0.0500 & \\
\hline & 2.2660 & 0.0210 & 0.0402 & 0.0520 & 0.0654 & 0.0700 & \\
\hline & 2.2660 & 0.0220 & 0.0232 & 0.0126 & 0.0720 & 0.0700 & \\
\hline & 2.2660 & 0.0230 & 0.0198 & 0.0711 & 0.0713 & 0.1000 & E \\
\hline ' & \multicolumn{6}{|l|}{$f_{x},>1$} & . \\
\hline
\end{tabular}

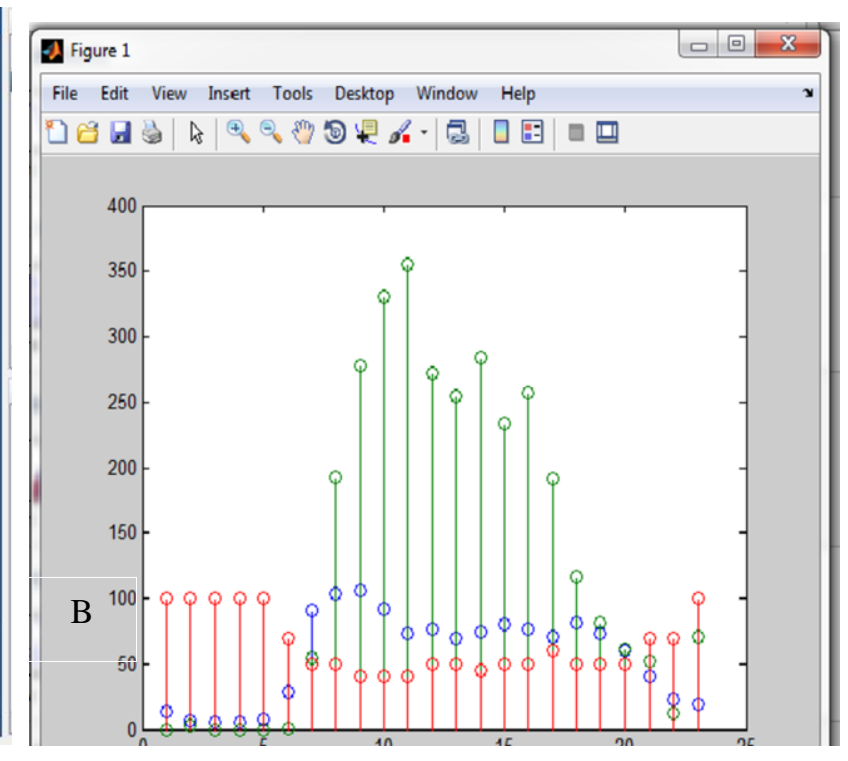

Fig. 3. Data analysis for square id 2266 and Comparison of speed limit with traffic flow and number of people. Figure $3 \mathrm{~A}$ shows the Matlab analysis output for square id 2266, first column represents square id, sec column indicates time (0 represents 00:00 to 01:00 and so on),third column represents traffic flow (its average number of vehicles in the particular square id, calculated based on relativity), fourth column is the approximate estimation of number of people based on CDR details(estimated based on relativity), fifth column is the normalized speed (median value) in that particular square id and last column is the speed limit estimated based on traffic and number of people. In Figure 3B red line indicates speed limit, blue indicates traffic flow and green for number of people. The speed limit is at maximum value 100 if traffic flow and people movement values are small and the value dropping down if number of vehicles and people movement increases

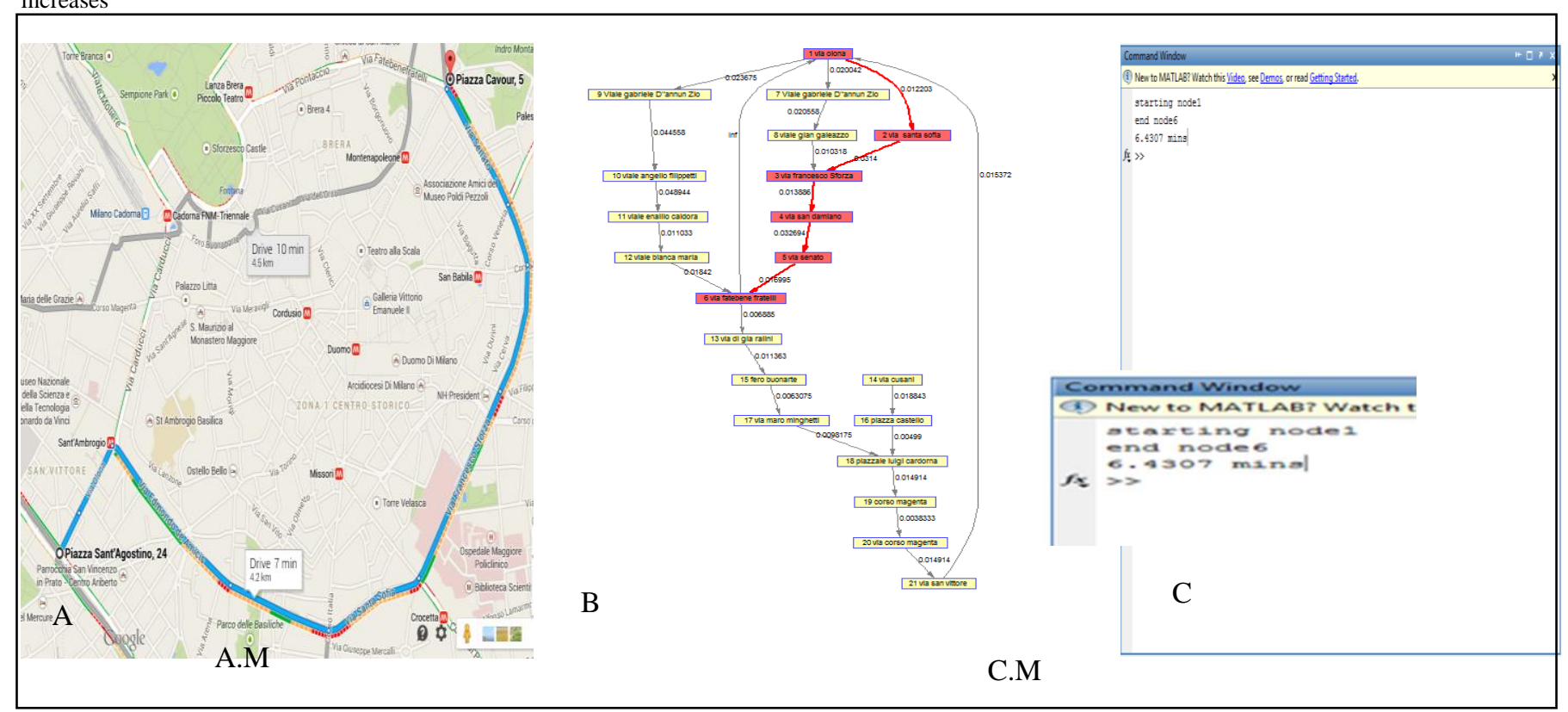

Fig. 4. Comparison between Google and the time estimation is shown. Figure 4.A shows google map shortest path and its estimated time taken, magnified image is shown in 4.A.M and Figure 4.B and 4.C shows the shortest path and time taken respectively, 4.C.M is the magnified version of 4.C. The Google predicted time is 7 mins between via olona and via Fatenbenefratelli and the model predicted time is 6.4307mins. Though the model is in early stages, GPS installed in car getting mandatory in future it improvises the accuracy of the time estimation 


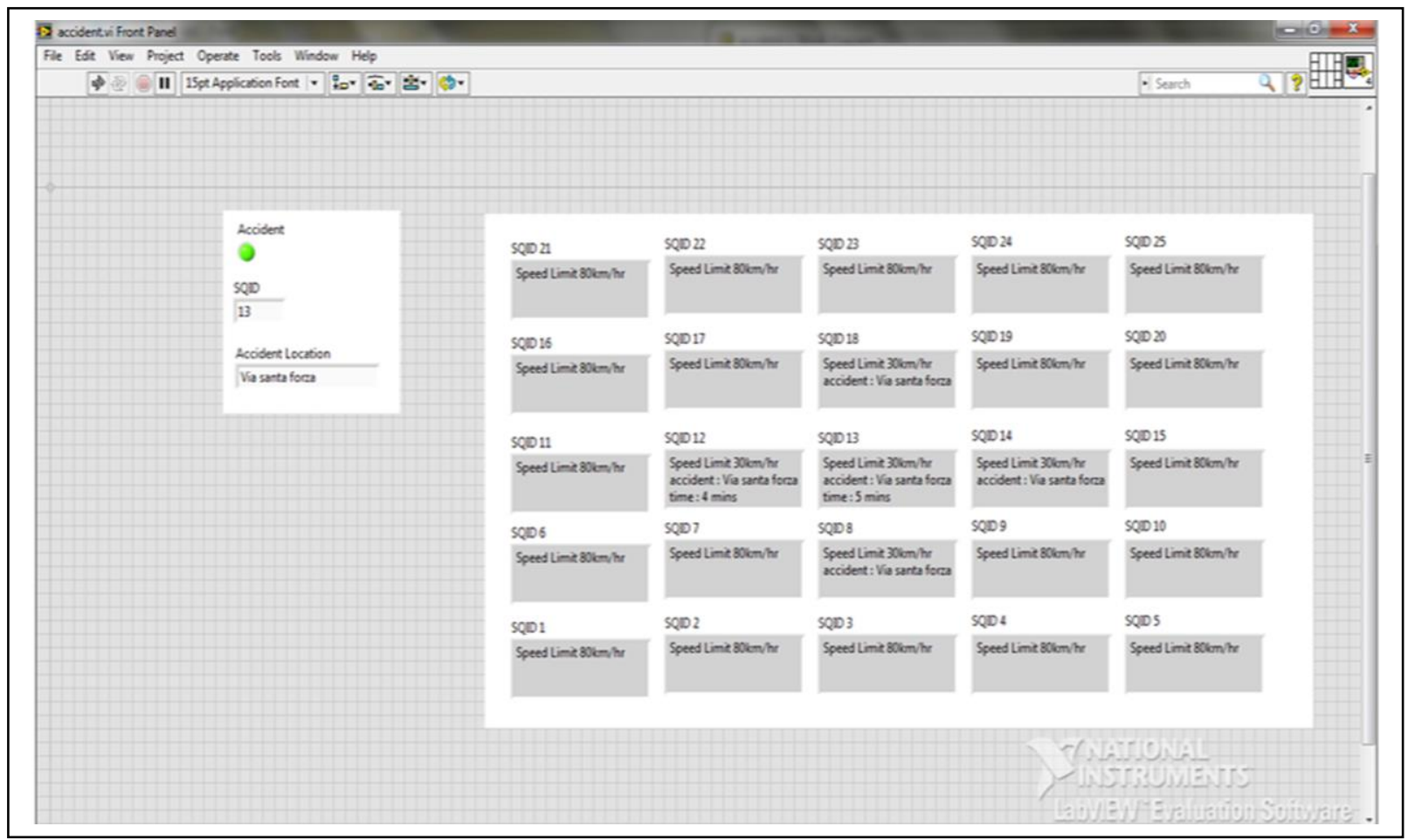

Fig. 5. Traffic congestion avoidance model during accident. Square id of $5 \times 5$ matrix is considered to depict the traffic avoidance congestion model during accident. The speed limit drops from $80 \mathrm{~km} / \mathrm{hr}$ to $30 \mathrm{~km} / \mathrm{hr}$ in accident spotted square id (13) and its neighbor square id (8, 12, 14 and 18), which controls the flow of the vehicle and reduces traffic snarl up. Adding to that if square id is located in the ambulance route then time taken for ambulance to cross the particular square id is displayed (square id 12 and 13). This would help ambulance to reach accident location on time

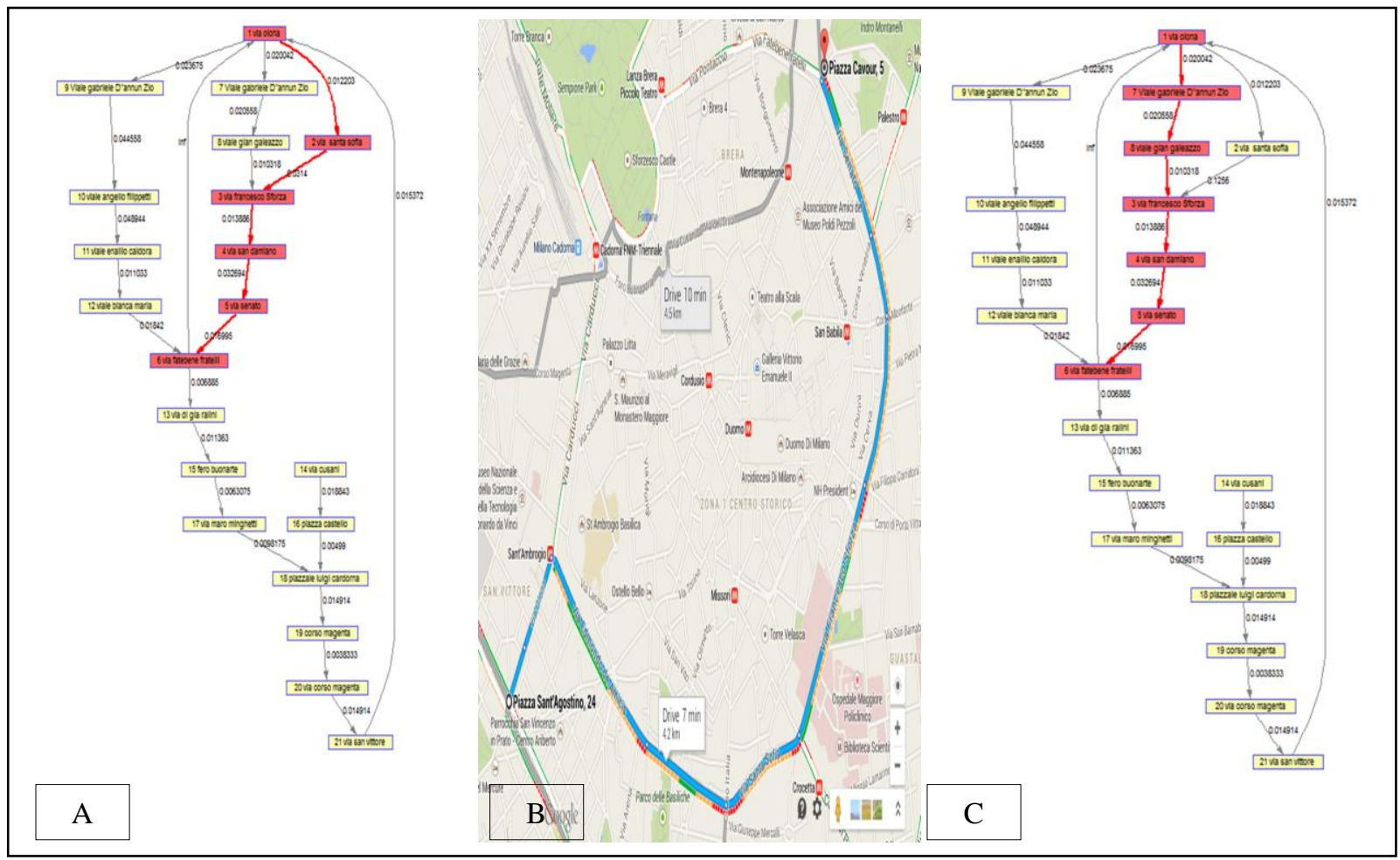

Fig. 6. Rerouted path during accident and when people movement increases drastically from normal in a particular square id. $6 . \mathrm{A}$ is the google hardest path between via Olona and via Fatenbenefratelli, 6.B is the shortest path between via Olona and via Fatenbenefratelli and the path taken is the same in both images proving the validity of the shortest path model and 6.C is the rerouted path when accident is spotted at via Santa Sofia, it reroutes the path via Viale Gabriele Dannum Zio and Viale Gian galeazzo to reach via Francesco Sforza instead of Santa Sofia 


\section{REFERENCES}

[1] Understanding the Networked Society: New Logics for an Age of Empowerment, Ericsson White Paper, UEN 284 23-3242, Feb, 2015 http://www.slideshare.net/Ericsson/wp-understandingthenetworked society

[2] Fred Mannering, Walter Kilareski and Scott Washburn, Principles of Highway Engineering and Traffic Analysis, Wiley-India, 3rd ed, 2005.

[3] Kyeong-Pyo Kang, Gang-Len Chang, and Nan Zou. "Optimal Dynamic Speed-Limit Control for Highway Work Zone Operations," Transportation Research Record: Journal of the Transportation Research Board, No. 187, TRB, National Research Council, Washington, D.C., 2004, pp. 77-84

[4] Lu Xutao, "Design of transport vehicles remote monitoring system," 2nd International conference on Education Technology and Computer (ICETC), Vol 2, pp. 310-313, 2010.

[5] M. Jason S. Claire, M. Stephen, and T. Denis, "3D laser imaging for surface roughness analysis”. International Journal of Rock Mechanics and Mining Sciences, Vol. 58, pp. 111-117, 2012

[6] Q. Li, M. Yao, X. Yao, and B. Xu, "A real-time 3D scanning system for pavement distortion inspection," Journal of Measurement Science and Technology, Vol. 21(1), pp. 15702-15709, 2010.

[7] Z. Hou, K. Wang, and W. Gong, "Experimentation of 3D pavement imaging through stereovision," Proceedings of International Conference on Transportation Engineering, pp. 376-381, 2007.

[8] X. Yu and E. Salari, "Pavement pothole detection and severity measurement using laser imaging," Proceedings of 2011 IEEE International Conference on Electro/Information Technology, pp. 1-5, 2011.

[9] K. Christian and B. Ioannis , "Pothole detection in asphalt pavement images." Journal of Advanced Engineering Informatics, Vol. 25 (3), pp. 507-515, 2011.

[10] B. Yu, and X. Yu, "Vibration-based system for pavement condition evaluation," Proceedings of the 9th International Conference on
Applications of Advanced Technology in Transportation, pp. 183-189, 2006.

[11] In Jung Lee, "An accident detection system on highway through CCTV with calogero-moser system," 18Th Asia pacific conference on Communication (APCC), pp. 522-525, 2012.

[12] C. Thompson. J. White. B. Dougherty, A. Albright, and D.C. Schmidt, "Using smart phones to detect car accident and provide situational awareness to emergence responders," $3^{\text {rd }}$ international ICST conference in Mobile wireless Middleware, Operating system and applications, Mobilware 2010, LNICST 48, pp. 29-42, 2010.

[13] M.S. Amin, J. Jalil, and M.B.I. Reaz, "Accident detection and reporting system using GPS, GPRS, and GSM technology," 2012 International conference on Informatics, Electronics and Vision (ICIEV), pp. 640643, 2012.

[14] J. Pan, M.A. Khan, I.S. Popa, K. Zeitouni and C. Borcea, "Proactive Vehicle Re-routing Strategies for Congestion Avoidance," 2012 IEEE $8^{\text {th }}$ international conference on Distributed Computing in Sensor Systems (DCOSS) pp. 265-272, ,2012.

[15] Siwei Jiang, Jie Zhang, and Yew-Soon Ong, "A Pheromone-based Traffic Management Model for Vehicle Re-routing and Traffic Light Control," Proceedings of the 2014 International Conference on Autonomous Agents and Multi-agent Systems, pp. 1479-1480, 2014.

[16] Lili Huang and Matthew Barth, "A Novel Loglinear Model for Freeway Travel Time Prediction," Proceedings of the 11th International IEEE Conference on Intelligent Transportation Systems, pp. 210-215, 2008.

[17] http://www.d4d.orange.com/en/home

[18] http://in.mathworks.com/products/matlab/

[19] http://www.ni.com/labview/

[20] http://in.mathworks.com/matlabcentral/fileexchange/27785-distance-cal culation -using-haversine-formula/content/haversine.m

[21] https://www.google.com/maps/d/viewer?mid=zNustSkyY4mQ.kbAGth cvLJu0\&ie=UTF8\&hq\&hnear=Italy\&source=embed\&oe=UTF\&\&showl $\mathrm{abs}=1 \& \mathrm{msa}=0$ 